\title{
Pilot study: peripheral biomarkers for diagnosing sporadic Parkinson's disease
}

\author{
Grünblatt, E ; Zehetmayer, S ; Jacob, C P ; Müller, T ; Jost, W H ; Riederer, P
}

\begin{abstract}
The need for an early and differential diagnosis of Parkinson's disease (PD) is undoubtedly one of the main quests of the century. An early biomarker would enable therapy to begin sooner and would, hopefully, slow or better prevent progression of the disease. We performed transcript profiling via quantitative RT-PCR in RNA originating from peripheral blood samples. The groups were de novo $(\mathrm{n}=11)$ and medicated PD $(\mathrm{n}=94)$ subjects and healthy controls $(\mathrm{n}=34)$, while for negative control Alzheimer's disease $(\mathrm{AD} ; \mathrm{n}=14)$ subjects were recruited as an additional neurodegenerative disease. The results were retested on a second recruitment consisting 22 medicated PD subjects versus 33 controls and $12 \mathrm{AD}$. Twelve transcripts were chosen as candidate genes, according to previous postmortem brain profiling. Multiple analyses resulted in four significant genes: proteasome (prosome, macropain) subunitalpha type-2 (PSMA2; p = 0.0002, OR = $1.1595 \%$ CI 1.07-1.24), laminin, beta-2 (laminin S) (LAMB2; $\mathrm{p}=0.0078, \mathrm{OR}=2.2695 \%$ CI 1.24-4.14), aldehyde dehydrogenase 1 family-member A1 (ALDH1A1; $\mathrm{p}=$ $0.016, \mathrm{OR}=1.0595 \% \mathrm{CI} 1.01-1.1)$, and histone cluster-1 H3e (HIST1H3E; $\mathrm{p}=0.03, \mathrm{OR}=0.97595 \% \mathrm{CI}$ 0.953-0.998) differentiating between medicated PD subjects versus controls. Using these four biomarkers for PD diagnosis, we achieved sensitivity and specificity of more than $80 \%$. These biomarkers might be specific for $\mathrm{PD}$ diagnosis, since in $\mathrm{AD}$ subjects no significant results were observed. In the second validation, three genes (PSMA2, LAMB2 and ALDH1A1) demonstrated high reproducibility. This result supports previous studies of gene expression profiling and may facilitate the development of biomarkers for early diagnosis of PD.
\end{abstract}

DOI: https://doi.org/10.1007/s00702-010-0509-1

Posted at the Zurich Open Repository and Archive, University of Zurich

ZORA URL: https://doi.org/10.5167/uzh-44559

Journal Article

Published Version

Originally published at:

Grünblatt, E; Zehetmayer, S; Jacob, C P; Müller, T; Jost, W H; Riederer, P (2010). Pilot study: peripheral biomarkers for diagnosing sporadic Parkinson's disease. Journal of Neural Transmission, 117(12):1387-1393.

DOI: https://doi.org/10.1007/s00702-010-0509-1 


\title{
Pilot study: peripheral biomarkers for diagnosing sporadic Parkinson's disease
}

\author{
Edna Grünblatt • Sonja Zehetmayer • \\ Christian P. Jacob • Thomas Müller • \\ Wolfgang H. Jost $\cdot$ Peter Riederer
}

Received: 22 July 2010/Accepted: 5 October 2010/Published online: 11 November 2010

(C) Springer-Verlag 2010

\begin{abstract}
The need for an early and differential diagnosis of Parkinson's disease (PD) is undoubtedly one of the main quests of the century. An early biomarker would enable therapy to begin sooner and would, hopefully, slow or better prevent progression of the disease. We performed transcript profiling via quantitative RT-PCR in RNA originating from peripheral blood samples. The groups were de novo $(n=11)$ and medicated PD $(n=94)$ subjects and healthy controls $(n=34)$, while for negative control Alzheimer's disease (AD; $n=14$ ) subjects were recruited as an additional neurodegenerative disease. The results were retested on a second recruitment consisting 22 medicated PD
\end{abstract}

Electronic supplementary material The online version of this article (doi:10.1007/s00702-010-0509-1) contains supplementary material, which is available to authorized users.

E. Grünblatt $(\square) \cdot$ C. P. Jacob · P. Riederer Clinical Neurochemistry, National Parkinson Foundation

Centre of Excellence Research Laboratories, Neurochemistry

Laboratory, Clinic and Policlinic for Psychiatry,

Psychosomatic and Psychotherapy, University of Würzburg,

Füchsleinstr 15, 97080 Würzburg, Germany

e-mail: edna.gruenblatt@kjpdzh.ch

\section{S. Zehetmayer}

Section for Medical Statistics, Medical University of Vienna,

Wien, Austria

T. Müller

Department of Neurology, St. Joseph Hospital,

Klinik-Weissensee, Berlin, Germany

W. H. Jost

Department of Neurology, Deutsche Klinik für Diagnostik, Aukammallee 33, 65191 Wiesbaden, Germany

E. Grünblatt

Department of Child and Adolescent Psychiatry,

University of Zürich, Zurich, Switzerland subjects versus 33 controls and $12 \mathrm{AD}$. Twelve transcripts were chosen as candidate genes, according to previous postmortem brain profiling. Multiple analyses resulted in four significant genes: proteasome (prosome, macropain) subunit-alpha type-2 (PSMA2; $p=0.0002$, OR $=1.15$ 95\% CI 1.07-1.24), laminin, beta-2 (laminin S) (LAMB2; $p=0.0078, \quad$ OR $=2.2695 \%$ CI 1.24-4.14), aldehyde dehydrogenase 1 family-member A1 (ALDH1A1; $p=$ $0.016, \mathrm{OR}=1.0595 \% \mathrm{CI} 1.01-1.1)$, and histone cluster-1 H3e (HIST1H3E; $p=0.03, \quad$ OR $=0.975 \quad 95 \% \quad$ CI 0.953-0.998) differentiating between medicated PD subjects versus controls. Using these four biomarkers for PD diagnosis, we achieved sensitivity and specificity of more than $80 \%$. These biomarkers might be specific for PD diagnosis, since in AD subjects no significant results were observed. In the second validation, three genes (PSMA2, LAMB2 and ALDH1A1) demonstrated high reproducibility. This result supports previous studies of gene expression profiling and may facilitate the development of biomarkers for early diagnosis of PD.

Keywords Aldehyde dehydrogenase 1 family, member A1 (ALDH1A1) · Biomarker · Blood · Diagnose/diagnosis . Gene expression - Histone cluster 1, H3E (HIST1H3E) Laminin beta 2 (LAMB2) - mRNA, Parkinson's disease (PD) · Proteasome (prosome, macropain) subunit, alpha type, 2 (PSMA2) - Transcriptome

\section{Introduction}

Parkinson's disease (PD) is a progressive neurodegenerative disorder, with a prevalence of $1 \%$ in the population aged above 65 years. The main pathophysiologic feature is degeneration of dopaminergic neurons in the substantia 
nigra, which results in striatal dopamine deficiency (Bernheimer et al. 1973). Nonetheless, the etiology of PD is still elusive.

Currently, PD patients are predominantly scored with the Unified Parkinson's Disease Rating Scale (UPDRS) (Fahn et al. 1987) and the modified Hoehn and Yahr staging scale (Hoehn and Yahr 1967). The criteria of the UK Brain Bank are employed for diagnosis (Hughes et al. 2002). Yet, even in highly specialized centers, the sensitivity of the clinical diagnosis of PD in symptomatic patients is only $91 \%$ and is likely to be lower in other settings (Tolosa et al. 2006). Familial forms of PD exist in about $5 \%$ of the affected population and involve mutations in many genes, e.g. $\alpha$-synuclein, LRRK2, UCLH-1, PINK1, DJ-1 and Parkin (Hatano et al. 2009; Klein et al. 2009). Functional imaging techniques, i.e. positron emission tomography with $\left[{ }^{18} \mathrm{~F}\right]$-Dopa or single photon emission tomography with $\left[{ }^{123} \mathrm{I}\right]-\beta$-CIT, visualize reduced striatal uptake of ${ }^{18} \mathrm{~F}$-DOPA in PD or loss of dopamine transporters, whereas for atypical PD variants the second option is limited (Brooks 1998; Coste et al. 2009; Koerts et al. 2007). Even these tools (clinical diagnosis and imaging) do not provide yet the specificity and sensitivity for PD diagnosis as one would wish for (Jankovic et al. 2000; Eerola et al. 2005; Kassiou et al. 2009). In addition, these diagnostic tools are only able to detect PD subjects after nearly $70 \%$ of the neurons have been degenerated, the point at which symptoms appear (Bhidayasiri 2006). This hampers treatment efforts to slow down degeneration or even rescue neurons. The importance of biomarkers for early diagnosis is discussed in many forms, especially as the case today is that by the time a patient is diagnosed with the disorder, the neurodegenerative process has been established for many years (Postuma and Montplaisir 2006; Paulsen 2009; Michell et al. 2004; Lovrecic et al. 2009; Mollenhauer and Trenkwalder 2009; Ravina et al. 2009). Hence, early diagnostic criteria are most important to allow an early therapeutic window.

In our previous study of gene expression profiles in postmortem brain tissue (Grünblatt et al. 2004), as well as four following studies (Hauser et al. 2005; Lu et al. 2005; Zhang et al. 2005; Simunovic et al. 2009), four pathways were shown to be altered in the substantia nigra of PD subjects: chaperones, ubiquitination, vesicle trafficking, and nuclear-encoded mitochondrial genes. Analysis of aberrant gene expression profiles in the central nervous system (CNS) of PD subjects cannot be accomplished during life. This problem may be circumvented by analyzing the peripheral tissues of PD subjects. Such an approach is recently discussed in PD as well as other movement disorders such as Huntington's disease (Lovrecic et al. 2009; Ravina et al. 2009). There is accumulating evidence of systemic alterations in key cellular functions of affected individuals (Scherzer et al. 2007; Sullivan et al. 2006; Hurley et al. 2003).

In the present study, we applied a similar strategy and extended our previous study in the CNS (Grünblatt et al. 2004) in an attempt to discover specific biomarkers for early diagnosis in blood. We used previous knowledge, obtained from brain postmortem gene expression profiling for PD, to choose candidate genes that might show patterns for risk scoring of PD in whole blood samples. Twelve potential transcripts were tested in early-non medicated (de novo), as well as medicate PD subjects and compared to healthy controls.

\section{Methods}

\section{Subjects}

The subjects gave informed consent according to the Helsinki Declaration. The study protocol was approved by the local ethics committee of the Universities of Würzburg, Bochum, and the Clinic of Wiesbaden, Germany. Written informed consent was obtained from all subjects or their primary caregivers. Patients with sporadic PD and healthy elderly controls without neurological disorders or dementia were assessed (excluded patients with familial PD by family anamnesis). As an additional control of other neurodegenerative disease, we recruited patients with Alzheimer's disease (AD) who did not suffer from any other neurological disorders. All subjects underwent formal diagnostic procedure according to the UK Brain Bank criteria for PD (Hughes et al. 2002). The Hoehn Yahr staging was used for clinical evaluation of PD (Hoehn and Yahr 1967) and the National Institute of Neurological and Communicative Disorders and Stroke-Alzheimer's Disease and Related Disorders Association criteria (McKhann et al. 1984) for AD. Alzheimer's disease assessment scalecognitive subscale (Wouters et al. 2008), mini-mental state examination (MMSE) (O'Connor et al. 1989), clinical dementia rating (CDR) (Berg 1988), UPDRS (Fahn et al. 1987), and Hamilton depression scale (Hamilton 1960) were administered to all subjects. In addition, detailed information on medication and smoking habits were collected. Some of the subjects were retested in a second recruitment after a period of 3-6 months from the first recruitment.

One hundred and fifty-three subjects (66 female and 85 male) with a mean age of $63.03 \pm 11.07$ years participated in the first recruitment (Table S1, Electronic Supplementary Material); 105 had PD (age $60.5 \pm 10.7$ years, MMSE scores $28.50 \pm 2.07$, UPDRS scores $31.34 \pm 18.82$, Hamilton depression scores $2.01 \pm 3.86$ ), 14 had AD (age $70.8 \pm 10.2$ years, MMSE scores $18.64 \pm 6.06$, UPDRS 
scores $9.36 \pm 10.40$, Hamilton depression scores $3.50 \pm$ 3.67 ) and 34 were healthy controls (age $67.6 \pm 9.8$ years, MMSE scores $29.47 \pm 1.13$, UPDRS scores $0.91 \pm 1.90$, Hamilton depression scores $3.68 \pm 6.79$ ). Of the 105 PD subjects, 11 were non-treated individuals, so called de novo PD subjects (age $55.7 \pm 11$ years, MMSE scores $29.2 \pm$ 0.87, UPDRS scores $27 \pm 7.7$, Hamilton depression scores $4.2 \pm 7)$. PD patients were treated with anti-parkinsonian standard therapy: with L-dopa and decarboxylase inhibitors as basic treatment. Sixty-seven subjects ( 37 female and 30 male) with mean of age $65.67 \pm 10$ years were reinvestigated in a second recruitment (Table S2, Electronic Supplementary Material), 22 had PD (age $61.4 \pm 9.3$ years, MMSE scores $28.68 \pm 1.21$, UPDRS scores $17.59 \pm$ 16.48, Hamilton depression scores $3.09 \pm 2.83$ ), 12 had $\mathrm{AD}$ (age $69.3 \pm 10.3$ years, MMSE scores $18.00 \pm 6.47$, UPDRS scores $8.67 \pm 10.33$, Hamilton depression scores $3.17 \pm 3.43$ ) and 33 were healthy controls (age $67.2 \pm$ 9.6 years, MMSE scores $29.55 \pm 0.94$, UPDRS scores $2.15 \pm 5.51$, Hamilton depression scores $3.33 \pm 6.11$ ). Whole blood was collected with the PAXgene ${ }^{\mathrm{TM}}$ Blood RNA system (Becton-Dickinson $\mathrm{GmbH}$, Heidelberg, Germany). The blood samples were frozen at $-20^{\circ} \mathrm{C}$ until further processing for total RNA isolation.

\section{Total RNA extraction}

Total RNA was prepared with the PAXgene ${ }^{\mathrm{TM}}$ Blood RNA Kit 50 (PreAnalytiX, Qiagen and BD, Germany). RNA isolation reagents were prepared from $0.2 \mu \mathrm{M}$ filtered, diethyl pyrocarbonate-treated water (Fermentas Inc., Hanover, MD, USA) throughout the isolation procedure. Total RNA samples were spectrophotometrically scanned (Experion, BioRad Co., Hercules, CA, USA) from 220 to $320 \mathrm{~nm}$; the $A 260 / A 280$ of total RNA was typically $>1.9$.

\section{Quantitative real-time RT-PCR analysis}

Quantitative real-time RT-PCR was conducted for 12 genes (Table S3, Electronic Supplementary Material): heat shock $70 \mathrm{kDa}$ protein 8 (HSPA8); proteasome (prosome, macropain) subunit, alpha type, 2 (PSMA2); proteasome (prosome, macropain) subunit, alpha type, 3 (PSMA3); proteasome (prosome, macropain) subunit, alpha type, 5 (PSMA5); heparan sulfate (glucosamine) 3-O-sulfotransferase 2 (HS3ST2); solute carrier family 31 (copper transporters), member 2 (SLC31A2); laminin, beta 2 (laminin S) (LAMB2); aldehyde dehydrogenase 1 family, member A1 (ALDH1A1); ubiquitin-conjugating enzyme E2K (UBC1 homolog, yeast) (HIP2); c-src tyrosine kinase (CSK); Cannabinoid receptor 2 (CNR2); Histone cluster 1, H3e (HIST1H3E); and 6 house-keeping genes (used as internal controls). Total RNA (500 ng) from each sample was reverse transcribed with the random hexamer and oligo-dT primer mix using iScript (BioRad Co., Hercules, CA, USA). Quantitative real-time PCR was performed in the iCycler iQ system (BioRad Co., Hercules, CA, USA) as described previously (Grünblatt et al. 2007). The genes were normalized to the six house-keeping genes according to GeNorm (Vandesompele et al. 2002). Real-time PCR was subjected to PCR amplification as described previously (Grünblatt et al. 2009). All PCR reactions were run in duplicate. The amplified transcripts were quantified using the comparative $C_{\mathrm{T}}$ method analyzed with the BioRad iCycler iQ system program. The same procedure was used for baseline samples as well as the follow-up confirmation study. Data were analyzed with Microsoft Excel 2000 to generate raw expression values.

Statistical analysis

For the first recruitment, mean, standard deviation (SD), median, minimum, and maximum values were calculated for the continuous variables (Table S1, Electronic Supplementary Material). In the text, continuous variables are described as mean \pm SD. For the data of the first recruitment, univariate logistic regression analyses were calculated for each of the 12 genes and the factors gender, age, CDR, MMSE, UPDRS, and Hamilton depression scale scores comparing the diagnosed PD subjects to healthy subjects. $p$ values, odds ratios (OR), their corresponding $95 \%$ confidence intervals $(95 \% \mathrm{CI})$, and the areas under the receiver operating characteristic curve (AUC) were calculated. Due to the small units the OR of the raw values are partly very large (e.g., OR $=1596047391$ ). Thus, we presented the ORs for the data as multiplied with 100 . All genes and co-variables (gender and age) with a $p$ value $<0.00357(0.05 / 14$ : Bonferroni adjustment for multiplicity) were further considered in a stepwise multiple logistic regression model. To avoid multicollinearity, a correlation of $R>0.6$ between two variables in the resulting model was not tolerated. The same approach was chosen for the analysis of AD versus healthy subjects (here, since no significant result was found in the logistic regression, no multiple model was calculated). Correlation analyses were performed between genes and the factors gender, age, CDR, MMSE, UPDRS and Hamilton depression scale scores. $p$ values $<0.008$ were considered significant (Bonferroni adjustment).

To investigate the validity of the gene measurements, Pearson correlation coefficients were calculated for the values of the first and the second recruitment (only for PD and healthy subjects). Intraclass correlation coefficients were calculated for the values of the first and second recruitment (for all groups). $p$ values $<0.0042$ were considered significant (Bonferroni adjustment). All computations 
were completed using the statistical computing environment $\mathrm{R}$ version 2.8 (http://www.r-project.org/, Department of Statistics and Mathematics of the WU Vienna, Austria) and SAS 9.1 (SAS Institute Inc., Cary, NC, USA).

\section{Results}

Logistic regression was conducted for all 12 genes and covariables for PD versus control subjects. The $p$ values, ORs (given for one hundredth of the measurements), and AUCs for the analysis of PD versus controls are presented in Table 1. The genes PSMA2, PSMA3, SLC31A2, LAMB2, ALDH1A1, and HIP2 significantly increased the risk for PD diagnosis, while HIST1H3E significantly decreased the risk for PD diagnosis. Increased UPDRS scores were significantly associated with increased risk of PD diagnosis.

All genes or risk factors with $p$ values $<0.00357$ were further selected for multiple analysis (genes, gender, and age). The model identified the following significant genes: PSMA2 $(p=0.0002, \quad$ OR $=1.1595 \%$ CI $1.07-1.24)$, LAMB2 $(p=0.0078, \quad$ OR $=2.2695 \%$ CI $1.24-4.14)$, ALDH1A1 $(p=0.016$, OR $=1.0595 \%$ CI 1.01-1.1), and HIST1H3E $(p=0.03$, OR $=0.97595 \%$ CI $0.953-0.998)$ for PD versus control. The receiver operating characteristic (ROC) curve was built with these four significant gene biomarkers (Fig. 1; $\max$ rescaled $R^{2}=0.62, \quad \mathrm{AUC}=$ 0.92). Using these four biomarkers for PD diagnosis, we achieved sensitivity and specificity of more than $80 \%$ (e.g.

Table 1 Logistic regression: PD versus control

\begin{tabular}{lllll}
\hline Gene & $p$ value & $\begin{array}{l}\text { Adjusted } \\
p \text { value }\end{array}$ & OR (95\% CI) & AUC \\
\hline HSPA8 & 0.014 & 0.24 & $1.04(1.01-1.07)$ & 0.674 \\
PSMA5 & 0.475 & 1 & $1.01(0.98-1.03)$ & 0.614 \\
PSMA2 & $2.68 \mathrm{E}-05$ & 0.00037 & $1.24(1.15-1.33)$ & 0.872 \\
PSMA3 & 0.002 & 0.028 & $1.06(1.02-1.1)$ & 0.714 \\
HS3ST2 & 0.505 & 1 & $1.08(0.86-1.36)$ & 0.524 \\
SLC31A2 & $2.00 \mathrm{E}-05$ & 0.00028 & $1.12(1.06-1.18)$ & 0.847 \\
LAMB2 & 0.00039 & 0.0055 & $2.54(1.52-4.26)$ & 0.825 \\
ALDH1A1 & $5.00 \mathrm{E}-05$ & 0.0007 & $1.07(1.03-1.1)$ & 0.758 \\
HIP2 & 0.00014 & 0.0020 & $1.05(1.03-1.08)$ & 0.743 \\
CSK & 0.004 & 0.056 & $1.03(1.01-1.06)$ & 0.742 \\
CNR2 & 0.231 & 1 & $0.99(0.97-1.01)$ & 0.546 \\
HIST1H3E & 0.001 & 0.014 & $0.97(0.96-0.99)$ & 0.72 \\
Gender & 0.013 & 0.182 & $2.76(1.24-6.14)$ & 0.624 \\
Age & 0.002 & 0.028 & $0.93(0.89-0.97)$ & 0.666 \\
\hline
\end{tabular}

For all parameters calculated in the regression, the OR refers to the higher value of the parameter. The adjusted $p$ value, is the Bonferroni corrected $p$ value, significance was set at $p<0.05$

$O R$ odds ratio, $C I$ confidence interval, $A U C$ area under the receiver operating characteristic curve, Gender females $=0$ and males $=1$
$91.5 \%$ sensitivity and $82 \%$ specificity). It must be noted that there are more than 50 possible models out of the set of univariate significant genes with an AUC larger than 0.87 and excluded correlations $>0.6$ between the variables in the model. All these models have similar sensitivity and specificity of more than $80 \%$.

Correlation analysis between the genes and the factors age, gender, MMSE, CDR, Hamilton depression scale, and UPDRS scores resulted in some significant correlations between the four genes (PSMA2, LAMB2, ALDH1A1, and HIST1H3E) and the parameters, age, MMSE, and UPDRS scores (Table 2; for all 12 genes see Tables S4 and S5, Electronic Supplementary Material). Increased age and UPDRS scores correlated significantly with the PSMA2 gene expression profile (decreased expression and increased expression, respectively). Nominal significance was found between MMSE scores and LAMB2 (increased expression with decreased score), age and ALDH1A1 (decreased expression with increased age), and UPDRS score and HIST1H3E (decreased expression with increased score).

Post hoc comparison of PSMA2, LAMB2, ALDH1A1, and HIST1H3E expression profiles between the 11 de novo PD subjects and the other 94 medicated PD subjects yielded a significant difference only for LAMB2; the expression profile of LAMB2 mRNA in the de novo PD subjects was lower than the medicated PD subjects ( $t$-test: $p<0.0001$; Table S5, Electronic Supplementary Material). Hoehn-Yahr scores (H\&Y) were slightly lower in de novo $\mathrm{PD}(\mathrm{H} \& \mathrm{Y}=2.00 \pm 0.50)$ subjects in comparison to the other PD subjects $(\mathrm{H} \& \mathrm{Y}=2.43 \pm 0.83$; Wilcoxon-test: $p=0.05$; Table S6, Electronic Supplementary Material).

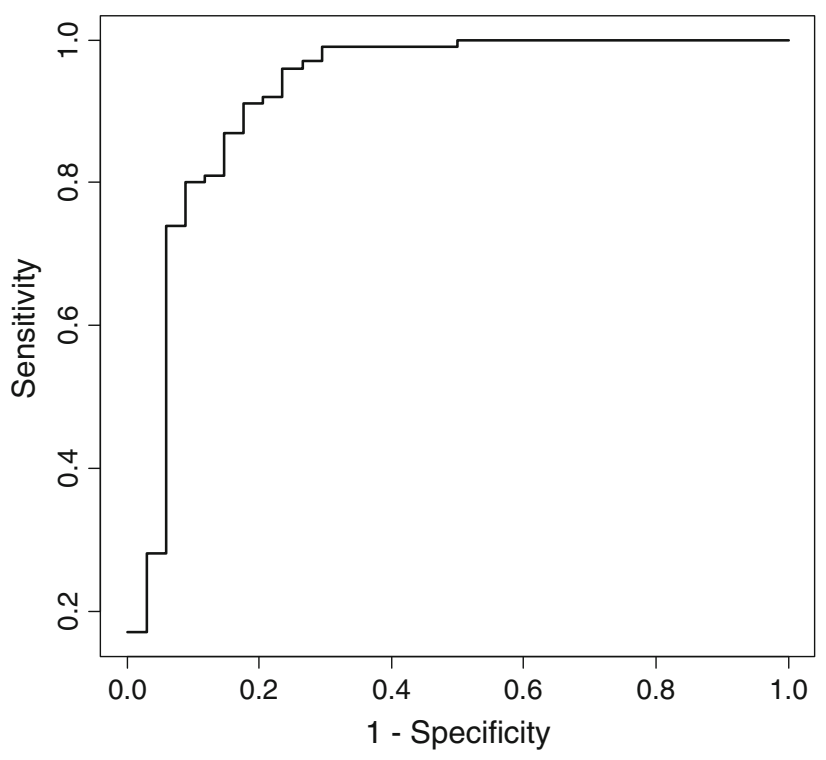

Fig. 1 ROC curve for the four biomarkers for PD diagnosis, PSMA2, LAMB2, ALDH1A1 and HIST1H3E 
Table 2 Correlations between genes and factors

\begin{tabular}{|c|c|c|c|c|}
\hline Gene & Factor & $\begin{array}{l}\text { Correlation } \\
\text { coefficient }\end{array}$ & $p$ value & $\begin{array}{l}\text { Adjusted } \\
p \text { value }\end{array}$ \\
\hline \multirow[t]{6}{*}{ PSMA2 } & Age & -0.274 & 0.001 & 0.006 \\
\hline & MMSE & -0.149 & 0.08 & 0.48 \\
\hline & UPDRS score & 0.331 & $<0.001$ & $<0.001$ \\
\hline & CDR score & -0.102 & 0.231 & 1 \\
\hline & Hamilton depression score & -0.186 & 0.059 & 0.354 \\
\hline & Gender & 0.035 & 0.258 & 1 \\
\hline \multirow[t]{6}{*}{ LAMB2 } & Age & -0.077 & 0.37 & 1 \\
\hline & MMSE & -0.235 & 0.005 & $\mathbf{0 . 0 3}$ \\
\hline & UPDRS score & 0.101 & 0.239 & 1 \\
\hline & CDR score & 0.157 & 0.065 & 0.36 \\
\hline & Hamilton depression score & 0.121 & 0.22 & 1 \\
\hline & Gender & 0.167 & 0.558 & 1 \\
\hline \multirow[t]{6}{*}{ ALDH1A1 } & Age & -0.238 & 0.005 & 0.03 \\
\hline & MMSE & 0.059 & 0.489 & 1 \\
\hline & UPDRS score & 0.12 & 0.16 & 0.96 \\
\hline & CDR score & -0.109 & 0.203 & 1 \\
\hline & Hamilton depression score & -0.045 & 0.647 & 1 \\
\hline & Gender & 0.159 & 0.066 & 0.396 \\
\hline \multirow[t]{6}{*}{ HIST1H3E } & Age & 0.15 & 0.084 & 0.504 \\
\hline & MMSE & 0.038 & 0.666 & 1 \\
\hline & UPDRS score & -0.189 & 0.029 & 0.175 \\
\hline & CDR score & 0.176 & 0.042 & 0.252 \\
\hline & Hamilton depression score & 0.012 & 0.905 & 1 \\
\hline & Gender & -0.005 & 0.53 & 1 \\
\hline
\end{tabular}

The adjusted $p$ value, is the Bonferroni corrected $p$ value, significance was set at $p<0.05$. Significant results are indicated in Bold obtain a measure for the reproducibility of the gene measurements conducted for the first recruitment. Some genes showed high reproducibility (Table 3; for intraclass correlation see Table S7, Electronic Supplementary Material), e.g., three genes from the multiple models for PD versus healthy (PSMA2, LAMB2, and ALDH1A1). Two genes showed only nominal significance for reproducibility (PSMA3 and CNR2), while two genes (HS3ST2 and HIST1H3E) had low reproducibility rates. For the second recruitment, a multiple model including the variables PSMA2, LAMB2, ALDH1A1, and HIST1H3E was used to calculate the AUC (max rescaled $R^{2}=0.66$, AUC $=0.93$ ) which resulted in sensitivity and specificity of more than $80 \%$.

\section{Discussion}

We found that the combination of four gene profiles in whole blood might be of diagnostic value for estimating the risk of developing sporadic PD. Since sporadic PD is a complex neurodegenerative disease, it is expected that a complex diagnostic tool with more than one marker is necessary (Fasano et al. 2008). Adding to the complexity of the disease classification, considerable overlap is seen at 
autopsy between patients clinically diagnosed with PD and those clinically diagnosed with other neurodegenerative diseases, i.e. sporadic AD (Grünblatt 2008).

As described by Fasano and colleagues (2008), as well as by Scherzer (2009), a multiple biomarker combination may be used for early diagnosis of PD. In the current study, we showed the combination of four biomarkers gave high sensitivity and specificity, which indicates the potential of such biomarkers to identify the risk of developing PD. Three of the genes were not influenced by PD medication, since no significant differences were observed between de novo PD subjects and medicated PD subjects. LAMB2 mRNA levels in de novo PD subjects were significant lower than treated PD subjects, while the expression was higher compared to controls; this might point to the disease progression, as well as treatment effects. One bias in the four biomarker model is the poor reproducibility of the HIST1H3E gene. But even when this gene is omitted and the three biomarkers are used in the multiple models, high specificity and sensitivity with AUC of 0.91 are found. Using multiple model analysis, we actually could find several models for biomarker batches that gave similar specificity and sensitivity. This might point to the complexity of such neurodegenerative diseases with regard to the cause of neurodegeneration and progress, as described by Hennecke and Scherzer (2008). Our choice of these four biomarkers for PD diagnosis is strengthened by the specificity to sporadic PD, as no significant association was found to link these biomarkers with sporadic AD subjects (Table S8, Electronic Supplementary Material).

Similar to our previous publication using microarray technology for a biomarker search in blood samples (Grünblatt et al. 2004), we showed a combination of gene expression profiles in cellular blood and linked clinical data can rapidly characterize candidate laboratory biomarkers of PD risk. Recently, Bogdanov et al. (2008) reported promising use of metabolomic blood biomarkers for PD diagnosis that may provide both diagnosis as well as monitoring disease progression in PD. Scherzer et al. (2007) described a biomarker search in whole blood from early stage PD subjects using microarray screening. In their study, they found eight biomarker genes that might be used as markers for risk scoring of PD. Out of the eight biomarkers, two similar genes were investigated in our study, the HIP and HSPA8 which is related to the ST13. Both genes significantly altered its expression in PD at the first and second recruitment, similar to Scherzers' report. Risk markers for complex diseases such as PD are not simply present or absent, rather they have a wide range of values that overlap in individuals (Manolio 2003). The risk typically increases progressively with rising levels. In our study, we searched for candidate genes based on our results from previous microarray studies with postmortem brain tissue (Grünblatt et al. 2004). We could not show any correlation when comparing the expression profile alterations between PD and controls in blood samples to postmortem tissue (Grünblatt et al. 2004). This does not indicate a flaw as transcription may be altered in peripheral blood cells by many factors such as copy number variations in the genome, epigenetic changes such as histone modifications, environmental changes causing biological processes such as mitochondria dysfunction, genetic and environmental changes, or as a response to brain pathology (Hennecke and Scherzer 2008).

In conclusion, we showed the potential of peripheral blood cell biomarkers as a tool for early diagnosis of PD. Further investigation in a larger independent population including different neurodegenerative disorders for differentiation is required also including familial analysis of different genetic backgrounds (e.g. glucocerebroside and $\alpha$-synuclein) to evaluate these biomarkers as potential diagnostic biomarkers as well as therapeutic targets for slowing the disease progression or even rescuing the neurons.

Acknowledgments This work was supported by the "Verein zur Durchführung neurowissenschaftliche Tagungen e.V" (2006) and the Hirnliga e.V. (2004). We wish to thank all the patients and the healthy volunteers who took part in this study and contributed to our findings. Special thanks to the study coordinator Monika Humann and the technicians, Miryame Hofmann and Carola Gagel, for their excellent work. All authors report no biomedical, financial, or potential conflicts of interest.

Conflict of interest All authors have no actual or potential conflicts of interest.

\section{References}

Berg L (1988) Clinical Dementia Rating (CDR). Psychopharmacol Bull 24:637-639

Bernheimer H, Birkmayer W, Hornykiewicz O, Jellinger K, Seitelberger F (1973) Brain dopamine and the syndromes of Parkinson and Huntington. Clinical, morphological and neurochemical correlations. J Neurol Sci 20:415-455

Bhidayasiri R (2006) How useful is (123I) beta-CIT SPECT in the diagnosis of Parkinson's disease? Rev Neurol Dis 3:19-22

Bogdanov M, Matson WR, Wang L, Matson T, Saunders-Pullman R, Bressman SS, Beal MF (2008) Metabolomic profiling to develop blood biomarkers for Parkinson's disease. Brain 131:389-396

Brooks DJ (1998) The early diagnosis of Parkinson's disease. Ann Neurol 44:S10-S18

Coste J, Ouchchane L, Sarry L, Derost P, Durif F, Gabrillargues J, Hemm S, Lemaire JJ (2009) New electrophysiological mapping combined with MRI in parkinsonian's subthalamic region. Eur J Neurosci 29:1627-1633

Eerola J, Tienari PJ, Kaakkola S, Nikkinen P, Launes J (2005) How useful is [123I]beta-CIT SPECT in clinical practice? J Neurol Neurosurg Psychiatry 76:1211-1216 
Fahn S, Elton R, Committee, Mot UD (1987) Unified Parkinson's disease rating scale. In: Fahn S, Marsden C, Goldstein M (eds) Recent developments in Parkinson's disease. Macmillan, New York, pp 153-167

Fasano M, Alberio T, Lopiano L (2008) Peripheral biomarkers of Parkinson's disease as early reporters of central neurodegeneration. Biomarkers Med 2:465-478

Grünblatt E (2008) Commonalities in the genetics of Alzheimer's disease and Parkinson's disease. Expert Rev Neurother 8:18651877

Grünblatt E, Mandel S, Jacob-Hirsch J et al (2004) Gene expression profiling of parkinsonian substantia nigra pars compacta; alterations in ubiquitin-proteasome, heat shock protein, iron and oxidative stress regulated proteins, cell adhesion/cellular matrix and vesicle trafficking genes. J Neural Transm 111:1543-1573

Grünblatt E, Zander N, Bartl J et al (2007) Comparison analysis of gene expression patterns between sporadic Alzheimer's and Parkinson's disease. J Alzheimers Dis 12:291-311

Grünblatt E, Bartl J, Zehetmayer S, Ringel TM, Bauer P, Riederer P, Jacob CP (2009) Gene expression as peripheral biomarkers for sporadic Alzheimer's disease. J Alzheimers Dis 16:627-634

Hamilton M (1960) A rating scale for depression. J Neurol Neurosurg Psychiatry 23:56-62

Hatano T, Kubo S, Sato S, Hattori N (2009) Pathogenesis of familial Parkinson's disease: new insights based on monogenic forms of Parkinson's disease. J Neurochem 111:1075-1093

Hauser MA, Li YJ, Xu H et al (2005) Expression profiling of substantia nigra in Parkinson disease, progressive supranuclear palsy, and frontotemporal dementia with parkinsonism. Arch Neurol 62:917-921

Hennecke G, Scherzer CR (2008) RNA biomarkers of Parkinson's disease: developing tools for novel therapies. Biomarkers Med 2:41-53

Hoehn MM, Yahr MD (1967) Parkinsonism: onset, progression and mortality. Neurology 17:427-442

Hughes AJ, Daniel SE, Ben-Shlomo Y, Lees AJ (2002) The accuracy of diagnosis of parkinsonian syndromes in a specialist movement disorder service. Brain 125:861-870

Hurley MJ, Mash DC, Jenner P (2003) Markers for dopaminergic neurotransmission in the cerebellum in normal individuals and patients with Parkinson's disease examined by RT-PCR. Eur J Neurosci 18:2668-2672

Jankovic J, Rajput AH, McDermott MP, Perl DP (2000) The evolution of diagnosis in early Parkinson disease. Parkinson Study Group. Arch Neurol 57:369-372

Kassiou M, Banati R, Holsinger RM, Meikle S (2009) Challenges in molecular imaging of Parkinson's disease: a brief overview. Brain Res Bull 78:105-108

Klein C, Schneider SA, Lang AE (2009) Hereditary parkinsonism: Parkinson disease look-alikes-an algorithm for clinicians to "PARK" genes and beyond. Mov Disord 24:2042-2058

Koerts J, Leenders KL, Koning M, Portman AT, van Beilen M (2007) Striatal dopaminergic activity (FDOPA-PET) associated with cognitive items of a depression scale (MADRS) in Parkinson's disease. Eur J Neurosci 25:3132-3136

Lovrecic L, Kastrin A, Kobal J, Pirtosek Z, Krainc D, Peterlin B (2009) Gene expression changes in blood as a putative biomarker for Huntington's disease. Mov Disord 24:2277-2281
Lu L, Neff F, Alvarez-Fischer D, Henze C, Xie Y, Oertel WH, Schlegel J, Hartmann A (2005) Gene expression profiling of Lewy body-bearing neurons in Parkinson's disease. Exp Neurol 195:27-39

Manolio T (2003) Novel risk markers and clinical practice. N Engl J Med 349:1587-1589

McKhann G, Drachman D, Folstein M, Katzman R, Price D, Stadlan EM (1984) Clinical diagnosis of Alzheimer's disease: report of the NINCDS-ADRDA Work Group under the auspices of Department of Health and Human Services Task Force on Alzheimer's disease. Neurology 34:939-944

Michell AW, Lewis SJG, Foltynie T, Barker RA (2004) Biomarkers and Parkinson's disease. Brain 127:1693-1705

Mollenhauer B, Trenkwalder C (2009) Neurochemical biomarkers in the differential diagnosis of movement disorders. Mov Disord 24:1411-1426

O'Connor DW, Pollitt PA, Hyde JB, Fellows JL, Miller ND, Brook CP, Reiss BB (1989) The reliability and validity of the minimental state in a British community survey. J Psychiatr Res 23:87-96

Paulsen JS (2009) Biomarkers to predict and track diseases. Lancet Neurol 8:776-777

Postuma RB, Montplaisir J (2006) Potential early markers of Parkinson's disease in idiopathic rapid-eye-movement sleep behaviour disorder. Lancet Neurol 5:552-553

Ravina B, Tanner C, Dieuliis D et al (2009) A longitudinal program for biomarker development in Parkinson's disease: a feasibility study. Mov Disord 24:2081-2090

Scherzer CR (2009) Chipping away at diagnostics for neurodegenerative diseases. Neurobiol Dis 35:148-156

Scherzer CR, Eklund AC, Morse LJ et al (2007) Molecular markers of early Parkinson's disease based on gene expression in blood. Proc Natl Acad Sci USA 104:955-960

Simunovic F, Yi M, Wang YL et al (2009) Gene expression profiling of substantia nigra dopamine neurons: further insights into Parkinson's disease pathology. Brain 132:1795-1809

Sullivan PF, Fan C, Perou CM (2006) Evaluating the comparability of gene expression in blood and brain. Am J Med Genet B Neuropsychiatr Genet 141:261-268

Tolosa E, Wenning G, Poewe W (2006) The diagnosis of Parkinson's disease. Lancet Neurol 5:75-86

Vandesompele J, De Preter K, Pattyn F, Poppe B, Van Roy N, De Paepe A, Speleman F (2002) Accurate normalization of real-time quantitative RT-PCR data by geometric averaging of multiple internal control genes. Genome Biol 3(7):research0034.1research0034.11

Wouters H, van Gool WA, Schmand B, Lindeboom R (2008) Revising the ADAS-cog for a more accurate assessment of cognitive impairment. Alzheimer Dis Assoc Disord 22(3):236244

Zhang Y, James M, Middleton FA, Davis RL (2005) Transcriptional analysis of multiple brain regions in Parkinson's disease supports the involvement of specific protein processing, energy metabolism, and signaling pathways, and suggests novel disease mechanisms. Am J Med Genet B Neuropsychiatr Genet $137: 5-16$ 